\title{
Fécondation assistée par micro injection des spermatides : Etat des lieux et questions posées
}

\author{
S. HamamaH*., C. Conord, J-M AYoubi**, N. Frydman \\ Centre de Fécondation in Vitro, Service de Biologie et Génétique de la Reproduction \\ Hôpital A. Béclère, 157 rue de la Porte-de-Trivaux 92141 Clamart \\ ** Service de Gynécologie-Obstétrique et de Médecine de la Reproduction, \\ CHU de Grenoble
}

\section{RESUME}

L'injection intracytoplasmique par ICSI d'un gamète immature (spermatide) a été réalisée dans plusieurs espèces animales (souris, hamster, lapin, et bovin), ainsi que chez l'homme. Le recours à des spermatides pour ICSI concerne des patients chez lesquels aucun spermatozoïde n'a été observé dans l'éjaculat ou dans le testicule. Vanderzwalmen et al (1995) rapportaient chez l'homme la fécondation d'ovocytes et le clivage embryonnaire par l'injection en ICSI de spermatides allongées. Cela a été confirmé par l'obtention de quelques naissances à la suite de l'injection ovocytaire de spermatides rondes ou allongées extraites de l'éjaculat ou du testicule. Mais, aujourd'hui, l'utilisation de spermatides en ICSI demeure très controversée. Le succès de l'ICSI avec une spermatide, son identification, ainsi que les problèmes posés, sont l'objet de cette revue.

Mots Clés: ICSI, spermatides, Fécondation, azoospermie

\section{INTRODUCTION}

Depuis 6 ans, nous avons vu se développer considérablement les moyens de lutte contre la stérilité masculine et ceci grâce à l'apparition de la technique de microinjection d'un spermatozoïde dans l'ovocyte (ICSI). L'avénement de cette technique a complétement révolutionné la prise en charge de la stérilité masculine. Elle a permis à des patients atteints d'oligozoospermie sèvère de devenir pères et pour lesquels la fécondation in vitro conventionnelle (FIVc) avait été peu efficace. Egalement, l'ICSI a permis à des patients azoospermiques, par la microinjection de spermatozoïdes épididymaires ou testiculaires, de réaliser leur projet de désir d'enfant.

Une nouvelle perspective depuis 1995 est apparue pour le traitement de patients ayant une spermatogénèse altérée, par l'injection de spermatides haploïdes ayant achevé leur méiose [30]. Le but de cette revue est (i) de faire un bilan des résultats de l'utilisation des spermatides en ICSI, chez l'homme ou chez l'animal, ainsi que des problèmes posés, et (ii) d'évaluer

* Correspondance: Pr. S. Hamamah Centre de Fécondation in Vitro, Service de Biologie et Génétique de la Reproduction Hôpital A. Béclère, 157 rue de la Portede-Trivaux 92141 Clamart 
le risque éventuel (génétique ou épigénétique) dû à l'injection intra -ovocytaire des spermatides dans le cadre d'un programme de thérapeutique de l'infertilité masculine.

\section{ICSI AVEC SPERMATIDE (EXPERIENCES ANIMALES)}

La première expérience publiée concernant la microinjection des spermatides date de 1993, finalement peu de temps après le développement de la technique de microinjection ellemême. Ogura et al (1993) [20]ont tenté de féconder des ovocytes matures de hamster en injectant le noyau de spermatides rondes. Ils montrent alors la capacité de ce noyau à se transformer en pronucleus mâle morphologiquement normal, synthétiser de l'ADN et se mélanger aux chromosomes de l'ovocyte pour participer à la syngamie. Un an après, ils obtiennent des embryons dont le transfert aboutit à la naissance de 4 souriceaux normaux (fertilité et comportement) [21]. D'autres naissances ont été obtenues chez le lapin [27], et chez la souris [17, 18], (voir Tableau 1). Dans ces travaux, les auteurs considèrent que les modifications postmitotiques de la spermatide (réorganisation du noyau, formation de l'acrosome et du flagelle), comme celles du spermatozoïde (maturation épididymaire, capacitation, réaction acrosomique), se développent uniquement pour permettre la pénétration du noyau haploïde mâle dans l'ovocyte.

\section{ICSI AVEC SPERMATIDE (RESULTATS CHEZ L'HOMME)}

Il s'agit de microinjecter un ovocyte avec une spermatide ronde, ou en début d'élongation, prélevée sur une biopsie testiculaire ou identifiée dans l'éjaculat de patients ayant une azoospermie $[30,40]$ (Tableau 2).

Que l'ICSI soit faite avec des spermatides rondes ou allongées, 12 enfants sont nés dont 3 avec spermatide ronde (ROSI), et 9 avec spermatide allongée (ELSI). Tesarik et al (1995) ont été les premiers a microinjecter des spermatides rondes provenant de l'éjaculat d'hommes dont l'azoospermie complète était inattendue le jour de la tentative de fécondation in vitro [30]. Sur les 7 transferts réalisés, 2 ont été suivis d'une grossesse puis une naissance à terme. La troisième naissance par ROSI a été publiée par Vanderzwalmen deux ans plus tard[41]. Les spermatides provenaient cette fois-ci de biopsies testiculaires.

Les taux de fécondation sont assez variables d'une équipe à l'autre. En effet, dans la majorité des cas, le taux de fécondation obtenu par microinjection de spermatides rondes est inférieur à celui classiquement obtenu par microinjection de spermatozoïdes. Une seule étude compare ces taux de fécondation sur une même cohorte ovocytaire[14]. L'ensemble des auteurs précisent que des améliorations techniques pourraient modifier les résultats. Le tableau 2 montre clairement que non seulement les taux d'implantation sont extrême-

Tableau 1 : Micro-injections de spermatides chez l'animal

\begin{tabular}{|c|c|c|c|c|}
\hline Espèce & Technique & $\begin{array}{l}\text { Embryons } \\
\text { transfèrés }\end{array}$ & Naissances & Reférences \\
\hline Souris & électrofusion de noyau & 346 & 4 & Ogura et al, 1994 [21] \\
\hline Lapin & injection de noyau & 121 & 3 & Sofikitis et al, 1994 [27] \\
\hline Souris & $\begin{array}{l}\text { injection de noyau } \\
\text { ou cellule entière }\end{array}$ & 131 & 35 & Kimura et al, 1995[17, 18] \\
\hline Lapin & injection de noyau & 150 & 14 & Sofikitis et al, 1996 [27] \\
\hline
\end{tabular}


ment bas, mais qu'on note également un nombre important de fausses couches spontanées. Deux équipes $[14,44]$ ont tenté de microinjecter non pas la cellule entière mais le noyau de la spermatide ronde (ROSNI: Round Spermatid Nucleus Injection). Les résultats étaient très prometteurs chez la souris [21]. L'injection du noyau permet d'utiliser une micropipette d'injection de diamètre inférieur à celui utilisé pour la cellule entière et diminue donc à priori le risque de léser l'ovocyte durant l'injection. Les auteurs ont pensé également que la persistance d'une grande quantité de cytoplasme autour du noyau pouvait gêner sa transformation en pronucleus mâle. Des embryons ont été obtenus et transférés mais aucune grossesse n'a été jusqu'à terme. On sait déjà que les ovocytes humains, contrairement aux ovocytes de souris, ne possèdent pas de centrosome, organite intracellulaire essentiel pour la division cellulaire. Ce dernier est fourni chez l'humain par le gamète mâle. De la même façon, le gamète mâle apporte un facteur d'activation ovocytaire appelé SSF (Sperm Soluble Factor ou oscilline) dont le rôle est important pour l'obtention d'embryons viables. D'après les résultats, il paraît préférable de ne pas se priver de l'ensemble des composants cytoplasmiques de la spermatide.

La première naissance par l'injection de spermatides allongées a été rapportée par Fishel et al (1995) [13]. Une biopsie testiculaire pratiquée chez le patient avait montré la présence de spermatozoïdes. Mais, le jour de la tentative, seuls 9 spermatozoïdes anormaux ont été retrouvés et l'auteur a préféré injecter les ovocytes avec des spermatides allongées (stade Sd1) de morphologie apparemment normale. Sur 10 ovocytes, un seul a été fécondé et a donné un embryon. Le transfert de cet embryon a abouti à une naissance. Un an après, Tesarik et al (1996) [32] obtiennent avec des spermatides allongées de l'éjaculat un taux de fécondation supérieur mais aucune grossesse. Sur un plus grand nombre de cas, les travaux de Kahraman et al (1997) [16], Antinori et al (1997) $[3,4]$ et Vanderzwalmen et al (1997) [41] montrent des taux de fécondation et de grossesse supérieurs pour l'injection de spermatides allongées par rapport à l'injection de spermatides rondes (Tableau 2).

Comment expliquer cette différence? Sur le plan théorique, les spermatides allongées représentent une étape plus avancée dans la spermatogenèse : la transition histone-protamine et la condensation de l'ADN ont déjà commencé, la maturité du cytoplasme et de ses composants (oscilline) est supérieure. Sur le plan technique, les spermatides allongées sont plus faciles à reconnaître que les spermatides rondes sous microscope optique. Enfin, leur taille permet l'utilisation de pipettes d'injection de diamètre identique à celles utilisées pour l'ICSI, contrairement aux spermatides rondes dont l'injection nécessite des pipettes de plus grand diamètre et présente donc un risque plus important de dommages ovocytaires [41]. Cependant, pour pouvoir comparer les résultats et évaluer le pouvoir fécondant des spermatides de différents stades de développement, il est important que l'ensemble des équipes adopte la même terminologie. De plus, dans la mesure où les classifications utilisées sont basées sur l'observation de spermiogenèses normales et dans la mesure où il n'existe pas encore de classification pour les formes pathologiques, il est nécessaire que les auteurs décrivent très précisément les stades utilisés [34, 35]. De plus, l'étude de Fishel et al (1997) [14] compare les taux de fécondation obtenus par injection de spermatozoïdes ou de spermatides rondes ou allongées chez 18 patients oligoasthénotératozoospermiques sévères ou ayant une azoospermie sécrétoire (tableau 3 ). Elle montre des taux de fécondation variant de 18 à $38 \%$ selon l'origine et le type des spermatides contre 63 à $74 \%$ pour les spermatozoïdes.

Le tableau 3 montre que les taux de fécondation varient selon l'origine des spermatides, et ceci inversement pour spermatides rondes ou allongées. Mais, il ne s'agit que de tendances, car statistiquement il n'y a pas de différences significatives. Cette étude confirme que dans tous les cas, quelles soient rondes ou allongées, provenant du testicule ou de l'éjaculat, les spermatides sont moins performantes que les spermatozoïdes. 


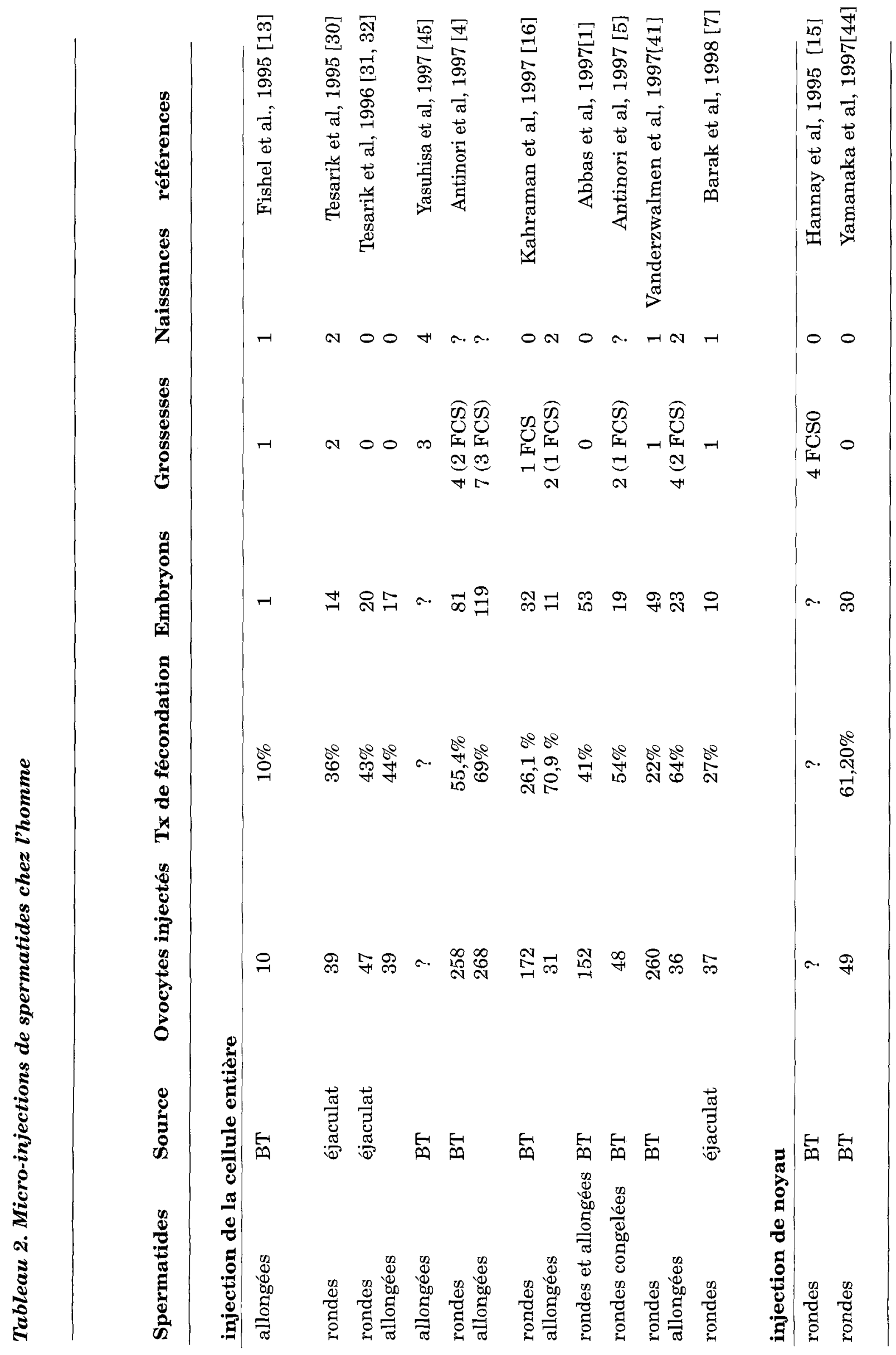


Tableau 3 : Comparaison des taux de fécondation obtenus par injection de spermatozoüdes ou de spermatides rondes ou allongées

\section{Source des gamètes}

\begin{tabular}{lcc} 
Cellule injectée & éjaculat & Testiculaire \\
\hline Spermatide ronde & $33 \%$ & $21 \%$ \\
Spermatide allongée & $18 \%$ & $38 \%$ \\
Spermatozoïdes & $63 \%$ & $74 \%$ \\
\hline
\end{tabular}

Fishel et al, 1997. [14]

\section{ASPECTS TECHNIQUES}

\section{Définitions}

ROSI : microinjection d'une spermatide ronde (Round Spermatid Injection) .

ROSNI : microinjection du noyau de la spermatide ronde (Round Spermatid Nucleus Injection).

ELSI : microinjection d'une spermatide allongée (Elongated Spermatid Injection).

\section{Identification d'une spermatide}

a) Description des stades successifs de différenciation

On distingue 6 types de spermatides en fonction de la forme de la cellule, de la forme du noyau et de l'aspect de la chromatine : (i) Sa et $\mathrm{Sb} 1$ : spermatides rondes, (ii) Sb2 et Sc : spermatides en élongation, et (iii) $\mathrm{Sd} 1$ et $\mathrm{Sd} 2$ : spermatides matures.

Sur frottis de sperme ou apposition de fragment de biopsie testiculaire fixés et colorés par la technique de Harris-Schorr, chacun de ces types de spermatide est reconnaissable. Ce n'est pas le cas sans coloration, donc sur les prélèvements frais utilisés pour la microinjection.

b) Comment reconnaître une spermatide ronde dans un prélèvement frais?

Lidentification et la sélection de la spermatide ronde est une étape fondamentale pour l'issue de la microinjection [44]. La spermatide se différencie des autres cellules rondes par sa taille, sa forme et les caractéristiques de son noyau. La spermatide ronde vivante présente un noyau rond central, entouré d'une zone régulière de cytoplasme. Les contours de la cellule sont lisses. Le diamètre varie de 6 à $8 \mu \mathrm{m}$, soit peu près celui d'un globule rouge[ 31,32 , $19,2,29]$. La taille de la spermatide est un critère de sélection important qui permet de différencier la spermatide ronde des spermatogonies, spermatocytes, polynucléaires et grands lymphocytes, cellules dont le diamètre est supérieur celui d'une spermatide ronde. Un granule acrosomique en développement est parfois visible sous la forme d'un spot de densité optique différente (brillant ou clair selon le système optique) adjacent au noyau. Ce critère, pourtant utile pour la sélection de la cellule, n'est pas toujours observé: les spermatides rondes précoces (phase Golgi du développement acrosomique) n'ont pas encore développé leur granule acrosomique alors que pour les stades les plus avancés, l'acrosome peut prendre la forme d'une cape sur la cellule, faiblement visible sur des cellules non colorées. Il faut donc savoir que la période pendant laquelle la vésicule acrosomique est clairement visible au sein de la spermatide ronde est limitée [42].

\section{c) Risques de confusion}

La spermatide ronde peut alors être confondue avec un petit lymphocyte, cellule dont la taille et la forme sont similaires. Il existe toutefois quelques différences: le lymphocyte présente un rapport nucléocytoplasmique plus élevé que la spermatide et le cytoplasme n'entoure pas le noyau de la cellule de façon régulière [2]. Silber et al [26] soulignaient récemment le risque de confusion entre spermatide ronde et noyau de cellule de Sertoli. Vanderzwalmen et al [42] et également Tesarik et al [36] s'opposent totalement à cette considération. Selon Tesarik, les noyaux des cellules de Sertoli sont beaucoup plus grands que les spermatides rondes et les restes cytoplasmiques qui les entourent parfois sont morphologiquement tout à fait différents du cytoplasme d'une spermatide ronde [36].

\section{d) Confirmation}

La méthode de sélection décrite repose uniquement sur des critères morphologiques dont l'observation est susceptible de varier d'un 
individu l'autre. Ainsi, Angelopoulos et al [2] a pu confirmer par FISH (Fluorescent In Situ Hybridation) que $80 \%$ des cellules rondes qu'il avait sélectionées au microscope inversé étaient bien des cellules haploïdes. De même pour Yamanaka [44] qui, après avoir sélectionné des spermatides rondes par analyse d'image assistée par ordinateur, a vérifié qu'il s'agissait de cellules haploïdes par microscopie électronique à transmission. D'autres méthodes de confirmation existent, comme l'utilisation d'un anticorps anti acrosine [31].

\section{RISQUES POTENTIELS}

Une des plus grandes craintes lors de l'introduction d'une nouvelle technique de conception assistée est celle de faire naître des enfants anormaux. Sur ce point, les premières informations concernant les enfants conçus par microinjection de spermatides sont plutôt rassurantes [30]. Mais le fait qu'un enfant soit normal à la naissance ne constitue pas la garantie d'un développement normal jusqu'à l'âge adulte. Dans le contexte d'application humaine, il existe deux questions fondamentales à poser: (i) pourquoi l'homme qui doit être inclus dans le programme de conception avec spermatides ne produit pas de spermatozoïdes ?; (ii) tous les éléments nécessaires au développement de l'embryon, outre le matériel génétique, sont-ils réunis dans les spermatides de ce patient?

\section{Facteurs Génétiques}

La stérilité sécrétoire, due à un déficit primaire de la production de spermatozoïdes, touche environ $5 \%$ des hommes infertiles [11]. L'existence de cas familiaux ou de remaniements chromosomiques montre qu'un défaut génétique peut être à l'origine de cette stérilité.

\section{A. Anomalies chromosomiques}

La comparaison entre les spermatozoïdes d'hommes fertiles et stériles montre une augmentation des anomalies de nombre et de structure des chromosomes. La fréquence globale de ces anomalies est inversement proportionnelle à la richesse en spermatozoïdes et varie dune étude à l'autre en fonction des cri- tères d'inclusion. Elle atteint près de $20 \%$ en cas d'azoospermie non obstructive, dont plus de $80 \%$ de caryotypes 47 , XXY, et 5-7\% environ en cas d'oligospermie légère, en majorité des anomalies des autosomes, translocations et inversions. Cette augmentation de fréquence est loin d'être négligeable et justifie en soi l'étude systématique du caryotype avant toute microinjection intraovocytaire d'une spermatide.

\section{B. Anomalies géniques}

Même si, dans la majorité des cas, l'origine de l'anomalie de spermatogenèse n'est pas identifiée, plusieurs régions du génome sont candidates et particulièrement sur le chromosome $Y$. Ce chromosome possède en effet plusieurs gènes impliqués dans la spermatogenèse.

\section{a)Description des anomalies de l'Y}

On savait depuis 1976, grâce la cytogénétique classique, que la perte du bras long du chromosome $\mathrm{Y}$ pouvait expliquer certaines azoospermies sécrétoires [39] et que des gènes contrôlant la spermatogenèse devaient être situés dans la partie non fluorescente, euchromatique, de ce chromosome, dans une région dénommée AZF pour Azoospermic Factor. Dans cette région, il a été identifié 3 loci appelés, $\mathrm{AZFa}, \mathrm{AZFb}$ et $\mathrm{AZF}$, dont la délétion serait responsable d'atteintes plus ou moins sévères de la spermatogenèse [43]. Il a été également identifié des gènes situés sur ces loci, les gènes YRRM1 et YRRM2 appartenant à une famille de gènes appelée YRRM pour " $Y$ chromosome genes with RNA recognition motif " et sur le locus le plus distal le gène DAZ pour "Deleted in Azoospermia " [25]. Vogt et al [43] ont évoqué la relation entre la région de l'Y manquante et le type d'atteinte de la spermatogenèse avec une gravité décroissante du locus AZFa au locus AZFc. Des microdélétions localisées en dehors des 3 sous-régions AZF peuvent être, elles aussi, associées à une infertilité masculine sévère et transmissible à la descendance. Il est même possible que le phénotype correspondant à une délétion dans le chromosome $\mathrm{Y}$ soit codéterminé par la présence et l'activité d'autres gènes contrôlant la spermatogenèse et localisés dans des autosomes, comme le gène DAZLA (DAZ Like 
Autosomal) récemment répéré sur le chromosome 3 .

\section{b) Fréquence des anomalies de l'Y}

La fréquence des anomalies de l'Y dans les infertilités masculines est difficile à déterminer. Car les études publiées sont différentes. Cette différence dans les résultats peut être expliquée en partie par les techniques utilisées et par les populations étudiées. Dans les azoospermies sécrétoires, la fréquence des anomalies de l'Y peut varier de 0 à $20 \%$ selon les études. En cas d'oligospermie sévère, la fréquence est plus faible et peut également varier de 0 à $10 \%$. Dans l'oligospermie moins sévère (> de $5 \times 10^{6}$ spermatozoïdes $/ \mathrm{ml}$ ), et dans la normospermie Pryor et al [23] observaient $2 \%$ de fréquence des anomalies de l'Y.

c) Conséquences pour la microinjection des spermatides

Il faut rappeler que toutes les expériences animales qui ont mené à la naissance d'une progéniture normale après utilisation de spermatides ont été réalisées chez des animaux fertiles. Il n'existe pas de modèle animal reproduisant les stérilités masculines susceptibles d'être traités par microinjection de spermatides [37]. Chez l'homme, comme on la vu, tout un ensemble de microdélétions sur le bras long du chromosome $Y$ peut être associé à une azoospermie sécrétoire, azoospermie avec parfois production de spermatides conservée [25]. Dès lors, comment savoir si ces spermatides sont génétiquement normales ? Si la procréation est rendue possible chez ces hommes par le biais de la conception avec spermatides, on peut craindre que l'infertilité du même type soit transmise à leur descendance masculine.

D'après les résultats des études publiées, ill faut distinguer 2 situations: (i) les techniques de ROSI et ELSI semblent donner de meilleurs résultats chez les patients pour lesquels une production de spermatozoïdes, même très limitée, a déjà été détectée [29,34,35,41], et (ii) le fait que le patient ait déjà démontré sa capacité à produire des spermatozoïdes matures, aussi peu nombreux soient-ils, permet d'exclure les anomalies génétiques les plus graves qui sont totalement incompatibles avec l'achèvement de la spermatogénèse. Une oligospermie très sévère alternant avec une azoospermie peut être provoquée par un grand nombre de facteurs environnementaux (infections virales, substances toxiques, traumatisme physique) et donc non transmissibles à la descendance. Limplication de facteurs non génétiques dans la pathogenèse d'une azoospermie est plus facilement envisageable lorsqu'une preuve existe que l'individu a pu produire des spermatozoïdes auparavant. C'est dans ces circonstances que la technique de ROSI a été utilisée avec succès pour la première fois [30, $31,32]$. En l'absence d'une telle preuve, la probabilité d'une étiologie génétique est sensiblement plus élevée. Les techniques de ROSI et ELSI réalisées chez des patients qui n'ont jamais montré de production spermatique donnent apparemment de moins bons résultats $[15,14]$.

A l'heure actuelle, le diagnostic d'une microdélétion $\mathrm{du}$ chromosome $\mathrm{Y}$, notamment lorsque celle-ci est importante et touche une des régions critiques, signifie un risque non négligeable de transmission d'hypofertilité voire d'infertilité dont la gravité est impossible à prévoir. Malgré un certain nombre d'incertitudes, cette recherche doit être effectuée en préalable d'une tentative de reproduction assistée chez tout homme atteint d'une oligospermie non obstructive sévère ou d'une azoospermie, associée au caryotype, afin de pouvoir conseiller le couple, tout en gardant en mémoire que l'ensemble des causes génétiques d'infertilité masculine n'est pas encore connu.

\section{FACTEURS EPIGENETIQUES}

Le spermatozoïde fécondant fournit à l' embryon non seulement le génome paternel mais aussi plusieurs facteurs épigénétiques. La contribution paternelle épigénétique comprend: (i) l'apport d'un facteur cytosolique responsable de l'activation ovocytaire, (ii) d'un centrosome fonctionnel et (iii) d'une empreinte spécifique sur certains gènes.

\section{a) Facteur d'activation ovocytaire}

L'activation ovocytaire chez les mammifêres implique notamment l'action d'un facteur spermatique cytosolique appelé SSF "Sperm 
Soluble Factor". Lors de la microinjection d'un spermatozoïde, la présence de ce facteur est indispensable pour activer l'ovocyte: Dorotzev et al [10] ont montré un effondrement des taux d'activation ovocytaire suite à l'injection de spermatozoïdes traités par la chaleur. Le SSF intervient certainement dans le déclenchement des oscillations calciques qui mènent à l'activation ovocytaire. Une injection directe de SSF dans l'ovocyte peut induire les oscillations calciques et l'activation ovocytaire. Souza al [28] a montré que l'injection d'une spermatide ronde dans l'ovocyte humain sensibilise l'ovocyte au développement d'oscillations calciques de façon similaire à l'injection d'un spermatozoïde mature et donc les spermatides rondes contiennent suffisamment d'activité SSF pour sensibiliser le mécanisme d'oscillations calciques de l'ovocyte humain. En plus, toute absence d'oscillations calciques typiques est probablement due à un déficit en SSF activité dans ces cellules. Cette étude montre que l'activité SSF se met en place entre le stade spermatocyte II et le stade spermatide ronde dans la spermatogenèse humaine normale. Une question reste sans réponse: qu'en est-il des spermatides rondes provenant d'hommes azoospermiques avec arrêt de maturation [29]?

\section{b) Centrosome}

Chez l'homme, le centrosome du spermatozoïde joue un rôle clé dans l'organisation microtubulaire durant la division cellulaire. On peut donc penser qu'en microinjection de spermatides, le centrosome paternel a déjà acquis la capacité d'orchestrer la division cellulaire du zygote au stade spermatide, avant la différenciation en spermatozoïde!

\section{c) Empreinte génomique}

L'empreinte génomique est une modification allèle-spécifique de l'ADN qui permet la reconnaissance et l'expression différentielle des allèles paternels et maternels de certains gènes. Une expression anormale de ces gènes, le plus souvent l'expression biallèlique ou l'absence d'expression au lieu d'une expression monoallèlique paternelle ou maternelle, est parfois associée aux pathologies humaines [24]. On évoque le cas d'un gène (snrpn) qui code pour un polypeptide du cerveau: les syndromes de Prader-Willi et d'Angelman seraient consécutifs, respectivement à la perte d'expression de l'allèle d'origine paternelle ou maternelle. Un cas isolé de gigantisme, associé à une tumeur rénale de Wilms, serait aussi dû à un défaut d'empreinte génomique, celle $d u$ gène Igf2.

L'empreinte génomique gamètique a lieu au cours de la gamétogénèse sans que l'on en connaisse le moment précis. Selon Testart [37], la naissance de souris normales et fertiles après microinjection de spermatides ou même de spermatocytes II indique que, dans cette espèce, l'empreinte génomique paternelle serait complète avant la spermiogénèse. Cependant, les données obtenues chez la souris indiquent que des modifications biochimiques liées à l'empreinte génomique (méthylation) ne sont pas achevées à l'issue du développement des spermatozoïdes dans le testicule mais continuent au cours du passage des spermatozoïdes dans l'épididyme [6]. La méthylation éventuelle de certains gènes pendant la formation ou la maturation du spermatozoïde paraît donc être en relation avec sa fonction immédiate dans la fécondation plutôt qu'avec sa fonction ultérieure dans le développement. De même, pour Fishel [12], les résultats obtenus chez la souris et chez l'homme montrent que l'empreinte génomique des cellules spermatogéniques se termine respectivement soit dans le testicule avant la 2ème division méiotique soit dans l'ovocyte mature après injection de la spermatide. On peut donc penser que tous les gènes imprimés ne le sont pas forcément en même temps au cours de la spermatogénèse. Ainsi, tous les gènes indispensables au développement jusqu'à la naissance pourraient être imprimés au stade spermatide ronde.

On peut cependant s'interroger sur le devenir des quelques enfants nés par conception avec cellules germinales immatures. Ils devraient faire l'objet d'un suivi particulier, avec notamment analyse de l'expression et de l'état de méthylation des gènes imprimés connus à l'heure actuelle. 


\section{CONCLUSION}

Malgré les incertitudes qui demeurent quant à l'efficacité de cette approche et les questions soulevées, quelques équipes dans le monde continuent à pratiquer la microinjection de spermatides rondes ou allongées. Pour Tesarik, désormais, pour les hommes concernés, il faut faire un choix entre deux attitudes distinctes: (i) continuer à prélever des échantions testiculaires pour trouver des spermatozoïdes au risque d'une destruction massive du tissu testiculaire, et (ii) pratiquer la microinjection de spermatides malgré une efficacité clinique très faible et des facteurs de risques potentiels supplémentaires [36]. Pour lui, choisir la technique de ROSI en alternative à l'lCSI avec spermatozoïdes testiculaires signifie une diminution du taux d'implantation de $50 \%$, mais diminue le risque de dommages testiculaires irréparables pour d'éventuelles futures tentatives qui auront lieu quand les efforts de recherche auront abouti à l'amélioration des taux de succès de ROSI. Devant les incertitudes, on doit plutôt se demander s'il est vraiment licite de considérer l'utilisation des spermatides comme une thérapeutique à part entière. Pour tout acte d'AMP et en particulier pour la microinjection de spermatides, les parents doivent être pleinement conscients des limites des connaissances actuelles, de la difficulté d'évaluer le risque qu'ils transmettent leur stérilité à leurs enfants, des conséquences à court terme et long terme. Il n'est pas certain qu'à l'heure actuelle, on puisse leur fournir tous les éléments dont ils auraient besoin pour prendre une décision.

A l'heure actuelle, le nombre de grossesses rapportées par cette approche est encore très faible et ne dépasse pas la dizaine d'enfants nés. Il n'est pas certain que l'utilisation de spermatides dans le programme d'ICSI ait grand avenir!

\section{REFERENCES}

1. ABBAS S.A., GHAZI S.A., SOLIMAN E.A., RAKHA A.M. : Experience with intracytoplasmic spermatid injection in the treatment of non obstructive azoospermia. 10th World Congress on in vitro fertilization and assisted reproduction, 1997, 609-613.

2. ANGELOPOULOS T., KREY L., MCCULLOUGH A., ADLER A., GRIFO J. : A simple and objective approach to identifying human round spermatids. Hum.Reprod., 1997,12, 2208-2216.

3. ANTINORI S., VERSACI C., DANI G., ANTINORI M., POZZA D., SELMAN H.A. : Fertilization with human testicular spermatids : four successful pregnancies. Hum. Reprod., 1997, 12, 286-291.

4. ANTINORI S., VERSACI C., DANI G., ANTINORI M., SELMAN H.A. : The use of round or elongated spermatids for ICSI : which is more effective ? 10th World Congress on in vitro fertilization and assisted reproduction, 1997, 547-551.

5. ANTINORI S., VERSACI C., DANI G., ANTINORI M., SELMAN H.A. : Successful fertilization and pregnancy using frozen-thawed round spermatids. 10th World Congress on in vitro fertilization and assisted reproduction, 1997, 699-703.

6. ARIEL M., CEDAR H., McCARREY J. : Developmental changes in methylation of spermatogenesis-specific genes include reprogramming in the epididymis. Nature Genetics, 1994, 7, 59-63.

7. BARAK Y., KOGOSOWSKI A; GOLDMA S., SOFFER Y., GONEN Y., TESARIK Y. : Pregnancy and birth transfer of embryos that developed from single nucleated zygotes obtained by injection of round spermatids into oocytes. Fertil Steril, 1998, 70, 67-70.

8. BUTLER D. : Spermatid injection fertilizes ethics debate. Nature, 1995, 377, 277.

9. DADOUNE J-P. : La spermatide, cette méconnue. Andrologie, 1997, 7, 22-23.

10. DOROTZEV D., RYBOUCHKIN A. DE SUTTER P. : Human oocyte activation following intracytoplasmic sperm injection : the role of the sperm cell. Hum. Reprod, 1995, 10, 403-407.

11. EDWARDS R.G., TARIN J.J., DEAN N., HIRSCH A., TAN S.L. : Are spermatid injections into human oocytes now mandatory ?. Hum.Reprod., 1994, 9, 2217-2219.

12. FISHEL S., ASLAM I., TESARIK J. : Spermatid conception : a stage too early, or a time too soon ?. Hum. Reprod., 1996, 11, 1371-1375.

13. FISHEL S., GREEN S., BISHOP M., THORNTON S., HUNTER A., FLEMING S., AL HASSAN S. : Pregnancy after intracytoplasmic injection of spermatid. The Lancet, 1995, 345, 1641- 1642.

14. FISHEL S., GREEN S., HUNTER A., LISI F., RINALDI L., LISI R., MCDERMOTT H. : Human fetilization with round and elongated spermatids. Hum. Reprod, 1997, 12, 336-340.

15. HANNAY T. : New japanese IVF method finaly made available in Japan. Nature Med., 1995, 1, 289-290.

16. KAHRAMAN S., POLAT G., SZEN E., ZGN O.D., DIRICAN K., ZBIER T., SAMLI M. : Multiple pre- 
gnancies obtained by testicular spermatid injection in combination with intracytoplasmic sperm injection. 10th World Congress on in vitro fertilization and assisted reproduction, 1997, 511-515.

17. KIMURA Y., YANAGIMACHI R. : Development of normal mice from oocytes injected with secondary spermatocyte nuclei. Biol.Reprod, 1995a, 53, 855-862.

18. KIMURA Y., YANAGAMACHI R. : Mouse oocytes injected with testicular spermatozoa or round spermatids can develop into normal offspring. Development, 1995b, 121, 2397-2405.

19. MENDOZA C., TESARIK J. : The occurrence and identification of rounds spermatids in the ejaculate of men with nonobstructive azoospermia. Fertil. Steril, 1996, 66, 826-829.

20. OGURA A., YANAGAMACHI R. : Round spermatid nuclei injected into hamster oocytes form pronuclei and participate in syngamy. Biol.Reprod, 1993, 48, 219-225.

21. OGURA A., MATSUDA J., YANAGIMACHI R. : Birth of normal young after electrofusion of mouse oocytes with round spermatids. Dev.Biol, 1994, 91, 7460-7462.

22. PLACHOT M. : La stérilité est-elle héréditaire ? Contracept. Fertil. Sex, 1997, 25, 194-195.

23. PRYOR J., KENT-FIRST M., MUALLEM A., VAN BERGEN A., NOLTEN W., MEISNER L., ROBERTS $\mathrm{K}$. : Microdeletions in the Y chromosome of infertile men. N. Engl. J. Med, 1997, 336, 534-539.

24. RAINIER S., JOHNSON L.A., DOBRY C.J. et coll. : Relaxation of impreted genes in human cancer. Nature, 1993, 362, 747-749.

25. REIJO R., LEE T.Y., SALO P et coll. : Diverse spermatogenetic defects in humans caused by $\mathrm{Y}$ chromosome deletions encompassing a novel RNA binding protein gene. Nature Genet., 1995, 10, 383-392.

26. SILBER S., JOHNSON L. : Are spermatid injections of any clinical value ?. Hum. Reprod, 1998, 13, 509515.

27. SOFIKITIS N., TODA T., MIYAGAWA I., ZAVOS P., PASYIANOS P., MASTELOU E. : Beneficial effects of electrical stimulation before round spermatid nuclei injections into rabbit oocytes on fertilization and subsequent embryonic development. Fertil.Steril, 1996, $65,176-185$.

28. SOUZA M., MENDOZA C., BARROS A., TESARIK J. : Calcium responses of human oocytes after intracytoplasmic injection of leukocytes, spermatocytes and round spermatids. Mol. Hum. Reprod., 1996, 2, 853857.

29. SOUZA M., BARROS A., TESARIK J. : Current problems with spermatid conception. Hum. Reprod., 1998, 13, 255-258.

30. TESARIK J., MENDOZA C., TESTART J. : Viable embryos from injection of round spermatids into oocytes, 1995, N. Engl. J. Med., 333, 525.
31. TESARIK J. , MENDOZA C. : Spermatid injection into human oocytes. I. Laboratory techniques and special features of zygote development. Hum.Reprod., 1996a, 11, 772-779.

32. TESARIK J., ROLET F., BRAMI C., SEDBON E., THOREL J., TIBI C., THEBAULT A. : Spermatid injection into human oocytes. II. Clinical application in the treatment of infertility due to non- obstructive azoospermia. Hum. Reprod, 1996b, 11, 780-783.

33. TESARIK J., MENDOZA C. : Genomic imprinting abnormalities : a new potential risk of assisted reproduction. Mol. Hum. Reprod., 1996c, 2, 295-298.

34. TESARIK J. : Use of immature spermatozoa and sperm precursor cells for fertilization. 10th World Congress on in vitro fertilization and assisted reproduction, 1997a, 661-667.

35. TESARIK J. : Sperm or spermatid conception. Fertil. Steril., 1997b, 68, 214-216.

36. TESARIK J., GRECO E., MENDOZA C. : ROSI, instructions for use : 1997 update. Hum. Reprod, 1998, $13,519-523$.

37. TESTART J. : De la spermatide au spermatozoïde : quels changements nécessaires au développement ?. Contracept. Fertil. Sex., 1996d, 24, 526-533.

38. THIBAULT C. : Certitudes et inquiétudes concernant ICSI. Contracept. Fertil. Sex, 1998, 26, 211-217.

39. TIEPOLO L., ZUFFARDI O. : Localization of factors controlling spermatogenesis in the nonfluorescent portion of the human Y chromosome long arm . Hum. Genet., 1976, 34, 119-124.

40. VANDERZWALMEN P., LEJEUNE B., NIJS M., SEGAL-BERTIN G., VANDAMME B., SCHOYSMAN R. : Fertilization of an oocyte microinseminated with a spermatid in an in-vitro fertilization programme. Hum.Reprod, 1995, 10, 502-503.

41. VANDERZWALMEN P., ZECH H., BIRKENFELD A., YEMINI M., BERTIN G., LEJEUNE B., NIJS M., SEGAL L., STECHER A., VANDAMME B., VAN ROOSENDAAL E., SCHOYSMAN R. : Intracytoplasmic injection of spermatids retrieved from testicular tissue : influence of testicular pathology, type of selected spermatids and oocyte activation. Hum. Reprod., 1997, 12, 1203-1213.

42. VANDERZWALMEN P., NIJS M., SCHOYSMAN R., BERTIN G., LEJEUNE B., VANDAMME B., KAHRAMAN S., ZECH H. : The problems of spermatid microinjection in the human : the need for an accurate morphological approach and selective methods for viable and normal cells. Hum. Reprod., 1998, 13, 515-519.

43. VOGT P.H., EDELMAN A., KIRSH S. et coll. : Human $\mathrm{Y}$ chromosome azoospermia factors (AZF) mapped to different subregions in Yq11. Hum. Mol. Genet., 1996, 5, 933-943.

44. YAMANAKA K., SOFOKITIS N, MIYAGAWA I., YAMAMOTO Y. and al. : Ooplasmic round spermatid injection procedures as an experimental treatement 
for non-obstructive azoospermia. J. of Ass. Reprod. and Gen., 1997, 14, 55-62.

45. YASUHISA A., MITSUHIRO M., ATSUMI Y., SANGYONG K., HIRO S., SHIGEO A. : Intracytoplasmic injection with late spermatids : a successful procedure in achieving childbirth for couples in which the male partner suffers from azoospermia due to deficient spermatogenesis. Fertil. Steril., 1997, 67, 559561.Tableau 1. Micro-injections de spermatides chez l'animal

\author{
ABSTRACT \\ ICSI WITH SPERMATID INJECTION : \\ a review and questions \\ S. Hamamah, C. Conord, J-M Ayoubi, \\ N. FRYDMaN
}

In order to treat male infertility, since 5 years ago, intracytoplasmic sperm injection (ICSI) represents a new therapeutic approach of different forms of male sterility including obstructive and spermatogenic failure azoospermia. This paper reviews the use of ICSI with round or elongated spermatids.

Key-words : ICSI, spermatids, fertilization, azoospermia 